\title{
156. On Extension of Continuous Mappings on Countably Paracompact Normal Spaces
}

\author{
By Kiyoshi IséKI \\ Kobe University \\ (Comm. by K. KUNUGI, M.J.A., Oct. 12, 1954)
}

The generalisations of extension theorem on continuous functions were developed by R. Arens (1), C. H. Dowker (5), J. Dugundji (6), and C. Kuratowski (12). A space is called countably paracompact if every countable open covering has a locally finite refinement. Hence countably paracompact spaces are a generalisation of paracompact spaces. C. H. Dowker and M. Katětov (11) have shown that countably paracompact normal spaces have many important properties. Recently B. J. Ball (2) proved that every linearly ordered space is countably paracompact.

In this note, we shall prove an extension theorem of continuous mappings on countably paracompact normal space, and a theorem of ANR as its application.

§1. An extension theorem on countably paracompact normal spaces

Theorem 1. Let $A$ be a closed subset of countably paracompact normal space $X$, and $f$ be a continuous map on $A$ with values in a separable Banach space $S$. Then $f$ may be extended to a map (continuous) $\bar{f}$ of $X$ into $S$.

The proof will be technically the same for the collectionwise normal space (C. H. Dowker (5)). We shall first some lemmas which will be used in the proof of Theorem 1 .

Lemma 1. Any Banach space is countably paracompact normal.

Proof. Since a metric space is paracompact normal (see A. H. Stone (15)), any Banach space is countably paracompact normal.

Lemma 2. If a is a locally finite covering of a normal space $X$, there is a canonical map $\phi$ of $X$ into the nerve $\left|X_{\alpha}\right|$ of $X$.

For the detail, see C. H. Dowker (4), p. 202 or S. Eilenberg and N. Steenrod (7), p. 286.

Proof (the idea only). Let $\beta$ be a shrinkable covering of $\alpha$. Since $X$ is normal, such a covering $\beta$ exists. (See S. Lefschetz (14), p. 26.) Therefore by the decomposition of unity, there are continuous maps $\phi_{\nu}(x)$ such that

1) $\phi_{\nu}(x) \geqslant 0$

2) $\sum \phi_{\nu}(x)=1 \quad$ for $x \in X$, 
3) $\phi_{\nu}(x)=0 \quad$ for $x \bar{\epsilon} U_{\nu} \in \alpha$.

Let $\phi(x)=\sum \phi_{\nu}(x) \cdot \nu$, where $\nu$ is the vertex corresponding to $U_{\nu} \in \alpha$, then $\phi(x)$ is a canonical map of $X$ into $\left|X_{\alpha}\right|$.

The proof of Theorem 1. Since $S$ is separable, the covering of $S$ by all open sets of diameter $\frac{1}{2^{n}}$ has a countable covering $\gamma_{n}$. Since $S$ is countably paracompact normal, $\gamma_{n}$ has star finite refinement $\beta_{n}$. (See K. Iséki (9), p. 350.) Therefore each $f^{-1}\left(\beta_{n}\right)$ is star finite countable open covering of $A$. Hence since a theorem of $\mathrm{O}$. Hanner, there is a locally finite open covering $\alpha_{n}$ of $X$ such that, for each $U$ of $\alpha_{n}, U \frown A$ is an element of $\alpha_{n}$.

Now we shall define a sequence of maps $\left\{f_{n}(x)\right\}$ by induction. If $n=1$ let $\alpha_{1}^{\prime}=\alpha_{1}$. Suppose that $n>1$, and $f_{n-1}: X \rightarrow S$ has been defined. Then $f_{n-1}^{-1}\left(\gamma_{n}\right)$ is a locally finite covering of $X$. Let $\alpha_{n}^{\prime}$ be a locally finite common refinement of $\alpha_{n}$ and $f_{n-1}^{-1}\left(\gamma_{n}\right)$. Let $\left|X_{\alpha^{\prime} n}\right|$ be the nerve by $\alpha_{n}^{\prime}$, and let $\phi_{n}$ be a canonical map of $X$ into $\left|X_{\alpha^{\prime} n}\right|$. By Lemma 2, such a map exists. For each $x$ of $X$, let $\Delta_{\alpha^{\prime} n}(x)$ be the simplex determined by $x$ in $\left|X_{\alpha^{\prime} n}\right|$. (See S. Eilenberg and N. Steenrod (7), p. 285.) Therefore $\phi_{n}(x) \in \Delta_{\alpha^{\prime} n}(x)$. We shall define a map $\psi_{n}:\left|X_{\alpha_{n}^{\prime}}\right| \rightarrow S$ as follows: Let $U$ be a set of $\alpha_{n}^{\prime \prime}$ and $u$ the corresponding vertex of $\left|X_{\alpha^{\prime}}\right|$. Let $U \frown A=0$, and if $n=1$, take any point of $S$ as the value of $\psi_{1}(u)$. If $n>1$, take $g_{n-1}(x)$ for a point $x$ of $U$ as $\psi_{1}(u)$. If $U \frown A \neq 0$, we shall define $\psi_{n}(u)$ by setting $\psi_{n}(u)=f(x)$ for fix $x \in U \frown A$.

$\psi_{n}(u)$ defined for the vertices of $\left|X_{\alpha^{\prime}}\right|$ may be extended linearly to a map $\psi_{n}:\left|X_{x^{\prime}}\right| \rightarrow S$. Therefore for $x \in X$, define $f_{n}(x)=\psi_{n}\left[\phi_{n}(x)\right]$, then $f_{n}(x)$ is clearly a continuous map of $X$ into $S$.

Let $n>1$ and $x, y \in U \in \alpha_{n}^{\prime}$, then

$$
\left\|f_{n-1}(x)-f_{n-1}(y)\right\|<\frac{1}{2^{n}} .
$$

1) If $U \frown A \neq 0, y \in A$, then

$$
\left\|f_{n-1}(y)-f(y)\right\|<\frac{1}{2^{n+1}}
$$

and by $\psi_{n}(u)=f(y)$,

$$
\left\|f_{n-1}(x)-\psi_{n}(u)\right\|<\frac{1}{2^{n-2}} .
$$

2) If $U \frown A \neq 0$, by $\psi_{n}(u)=f_{n-1}(y)$, then

$$
\left\|f_{n-1}(x)-\psi_{n}(u)\right\|<\frac{1}{2^{n}}<\frac{1}{2^{n-2}} .
$$

Therefore we have

$$
\left\|f_{n-1}(x)-\psi_{n}\left[\phi_{n}(x)\right]\right\|<\frac{1}{2^{n-2}} .
$$


Hence

$$
\left\|f_{n-1}(x)-f_{n}(x)\right\|<\frac{1}{2^{n-2}} .
$$

Let $U \frown A \neq 0$ and $\psi_{n}(u)=f(y)$ for $y \in U \frown A$, then for $x \in U \frown A$ we have

$$
\left\|f(x)-\psi_{n}(u)\right\|<\frac{1}{2^{n}}
$$

Therefore

$$
\left\|f(x)-\psi_{n}\left[\phi_{n}(x)\right]\right\|<\frac{1}{2^{n}} .
$$

This shows that $\left\|f(x)-f_{n}(x)\right\|<\frac{1}{2^{n}}$. The maps $f_{n}(x): X \rightarrow S \quad(n=$ $1,2,3 \ldots)$ satisfy the conditions:

$$
\begin{gathered}
\left\|f_{n}(x)-f_{n-1}(x)\right\|<\frac{1}{2^{n-2}}, \\
\left\|f_{n}(x)-f(x)\right\|<\frac{1}{2^{n}} \text { for } x \in A .
\end{gathered}
$$

Since $f_{n}(x)$ is convergent in norm, the limit map $\overline{f(x)}$ is continuous and

$$
\left\|f_{n}(x)-\bar{f}(x)\right\| \leq \frac{1}{2^{n-2}}
$$

If $x \in A$, then

$$
\begin{gathered}
\|f(x)-\bar{f}(x)\| \leq\left\|f(x)-f_{n}(x)\right\|+\left\|f_{n}(x)-\bar{f}(x)\right\| \\
\leq \frac{1}{2^{n-3}} .
\end{gathered}
$$

This shows that $f(x)=\bar{f}(x)$ for $x \in A$, and $\bar{f}(x)$ is an extension map of $f(x)$. Q. E. D.

A method similar to Theorem 1 or the results of C. H. Dowker (5) and R. H. Bing ((3), p. 183) implies the following.

Theorem 2. Let $A$ be a closed subset of a paracompact normal spaces $X$ and $f$ be continuous on $A$ with values in a Banach space $S$. Then $f$ may be extended to a map $\bar{f}$ of $X$ into $S$.

$\S 2$. A theorem on separable metric ANR (countably paracompact normal)

In this section, we shall prove, as an application of Theorem 1:

Theorem 3. A separable metric space is ANR (countably paracompact normal) if and only if it is ANR (separable metric) and toplogically complete.

Proof. The necessity of the condition follows from one of my results. (See K. Iséki (10), p. 571.) 
Conversely, let $Y$ be an ANR (separable metric), and topologically complete, i.e. absolute $G_{\delta}$. By a well-known theorem of C. Kuratowski, K. Kunugi, and M. Wojdyslawski (see C. Kuratowski (13), p. 112), Y is a subspace of a separable Banach space $S$. To prove that $Y$ is an ANR (countably paracompact normal) let $X$ be a countably paracompact normal space, $A$ a closed subset of $X$ and $f: A \rightarrow Y$ a map of $A$ into $Y$. By Theorem 1, we have an extension $f_{1}: X \rightarrow S$ of $f$. Since $Y$ is $G_{\delta}$-set in $S$, we can find open sets $G_{n}(n=1,2,3, \ldots)$ of $S$ such that $Y=\bigcap_{n} G_{n} \cdot f_{\mathrm{I}}^{-1}\left(G_{n}\right)(n=1,2,3, \ldots)$ are open in $X$ and contain the closed set $A$. Therefore there are continuous maps $\phi_{n}(x)$ on $X$ such that

1) $0 \leq \phi_{n}(x) \leq 1$,

2) $\phi_{n}(x)=0$ on $A$,

3) $\phi_{n}(x)=1$ on $X-f_{1}^{-1}\left(G_{n}\right)$.

The map

$$
\phi(x)=\sum_{n} \frac{1}{2^{n}} \phi_{n}(x)
$$

is continuous on $X, \phi(x)=0$ on $A$, and $\phi(x)>0$ if $f_{1}(x) \in S-Y$. Then $f_{2}(x)=\left(f_{1}(x), \phi(x)\right)$ is continuous on $X$ into $S^{\prime}=S \times I-(S-Y) \times 0$, where $I=\{t \mid 0 \leq t \leq 1\}$. The map $\Psi: Y \times 0 \rightarrow Y$ defined by $\Psi(y, 0)=Y$ is extendable to a neighbourhood $V$ of $Y \times 0$, since $Y \times 0$ is closed in the metric space $S^{\prime}$ and $Y$ is ANR (metric). For its extension map $\bar{\Psi}$, let $U=\bar{\Psi}^{-1}(V)$, then $U$ is a neighbourhood of $A$ in $X$. Define $\bar{f}: U \rightarrow Y$ by $\vec{f}(x)=\bar{\Psi}\left[f_{2}(x)\right]$, then $\bar{f}$ is an extension of $f$ and a retraction of $U$ onto $Y$. This shows that $Y$ is ANR (countably paracompact normal). Q. E. D.

From Theorem 3 and a result of 0 . Hanner ((8), p. 378), we have the following.

Theorem 4. A separable metric space is ANR (countably paracompact normal) if and only if it is ANR (normal).

\section{References}

1) R. Arens: Extension of functions on fully normal spaces, Pacific Jour. Math., 2, 11-22 (1952).

2) B. J. Ball: Countable paracompactness in linearly ordered spaces, Proc. Am. Math. Soc., 5, 190-192 (1954).

3) R. H. Bing: Metrisation of topological spaces, Canadian Jour. Math., 3, 175-186 (1951).

4) C. H. Dowker: Mapping theorems for non-compact spaces, Amer. Jour. Math., 69, 200-242 (1947).

5) C. H. Dowker: On a theorem of Hanner, Ark. Mat., 2, 307-313 (1952).

6) J. Dugundji: An extension of Tietze's theorem, Pacific Jour. Math., 1, 353-368 (1951). 
7) S. Eilenberg and N. Steenrod: Foundations of algebraic topology. Princeton (1952).

8) O. Hanner: Solid spaces and absolute retracts, Ark. Mat., 1, 375-382 (1951).

9) K. Iséki: A note on countably paracompact spaces, Proc. Japan Acad., 30, 350-351 (1954).

10) K. Iséki: On a property of mappings of metric spaces, Proc. Japan Acad., 30, 570-571 (1954).

11) M. Katětov: On real valued functions in topological spaces, Fund. Math., 38, 85-91 (1951).

12) C. Kuratowski: Sur quelques problèmes topologiques concernant le prolongement des fonctions continues, Coll. Math., 2, 186-191 (1951).

13) C. Kuratowski: Topologie, 1 (3rd edition). Warszawa (1952).

14) S. Lefschetz: Algebraic topology. Princeton (1942).

15) A. H. Stone: Paracompactness and product spaces, Bull. Am. Math. Soc., 54, 977-982 (1948). 